\title{
THE EFFECT OF TOPICAL MEDICATION CONTAININGBENZALKONIUMCHLORIDE ON OCULAR SURFACE DISEASE INGLAUCOMA PATIENTS
}

\author{
${ }^{* 1}$ Nurwasis, ${ }^{1}$ Rina Wulandari, ${ }^{1}$ Delfitri Lutfi
}

ABSTRACT---Background, The main cause of the emergence of dry eye and ocular surface disease (OSD) is strongly suspected due to the preservative component of topical medication in the form of benzalkonium chloride(BAK).Various complaints such as uncomfortable, teary, redness, burning and foreign object sensation, glare, often blinking and intermittent blurred vision has-been reported as part of OSD.

Objective: The study aimed to investigate the effect of topical medication containing BAK on ocular OSD in glaucoma patients

Method: Subject who has been using topical antiglaucomatous medication containing BAK and artificial tears were examined for tear film break up time (TBUT), S I test and corneal staining. Sucject were allowed to close the eyes, then positioned in front of the slit-lamp biomicroscope. Regression test was used to analyze the correlation between each variable.

Result: TBUT and S I test were decreased, and corneal staining score was increased, furthermore, BAK is significantly correlated with all of the examination result by using regression test. The effect of the amount and duration of BAK used is significantly correlated with TBUT $(p=0.004)$. The effect of the amount and duration of $B A K$ used insignificantly correlated with $S$ I test $(p=0.043)$. This study also has $R$ value as 0.901 ; >0.05, shows the amount and duration of BAK used is strongly affect TBUT, S I test and corneal staining score.

Conclusion: BAK has strong correlation on the event of ocular surface disease.

Keywords---benzalkonium chloride, glaucoma, ocular surface disease, Schirmer I test, corneal staining.

\section{INTRODUCTION}

Glaucoma is reportedly the leading cause of irreversible blindness in the world, affecting $>70$ million people worldwide(Leung, Medeiros and Weinreb, 2008).This disease is caused by a group of optic neuropathies that lead to progressive degeneration of retinal ganglion cells. Damage to these neurons results in what is called "cupping" of the optic disk and vision loss(Halasz et al., 2019).The main treatment of glaucoma is the use of topical medication, and often can't be treated with a single treatment. In a data from the use of topical medication in patients with

\footnotetext{
${ }^{1}$ Department of Ophthalmology, Faculty of Medicine, Airlangga University/ Dr. SoetomoGeneral Hospital, Surabaya 60131, Indonesia *Coresponding Author: Dr.Nurwasis dr., Sp.M(K)

Ophthamology Department, Medical Faculty of Airlangga University, Surabaya 60131, Indonesia.

Email: nurwasisspm@yahoo.com; nurwasis.unair@gmail.com
} 
International Journal of Psychosocial Rehabilitation, Vol. 24, Issue 02, 2020

ISSN: $1475-7192$

glaucoma in the United States, 49-59\% of patients also have dry eyes and ocular surface disease (OSD)(Zhang et al., 2019).

The main cause of the emergence of dry eye and OSD is strongly suspected due to the preservative component of topical medication in the form of benzalkonium chloride(BAK). This additional preservative also functions as bactericidal and increases drug penetration, but can have an unfavorable effect, causing additional complaints and OSD which affecting therapy compliance (Zhang et al., 2019).

Various complaints such as uncomfortable, teary, redness, burning and foreign object sensation, glare, oftenly blinking and intermittent blurred vision has been reported as part of OSD. This occurs due to disruption in the tear, conjunctiva land corneal upper layer with the sign of reduced tear film break up time (TBUT)and Schirmer I test (S I test), also the present of corneal staining(Moss, Klein and Klein, 2000; Kaštelan et al., 2013; Amparo, Schaumberg and Dana, 2015).According to the facts, this study aim is to investigate the effect of topical medication containing BAK on ocular OSD in glaucoma patients.

\section{METHODS}

This is a cross sectional observational analytic study in the glaucoma division outpatient clinic of Department of Ophthalmology, dr. Soetomo General Hospital Surabaya. This study been held in one month, August 2019. Each subject recruitment and variable examination were conducted at the same day. Ethical clearance was conducted from Ethical Committee Dr.Soetomo Hospital, Surabaya.

After been informed and sign a consent of study. One of the eye were evaluated in order of S I test, TBUT and corneal staining examination. Firstly, without topical anesthesia, Schirmer paper was placed in inferior fornix at lateral area, then after $5 \mathrm{~mm}$, data we recollected in $\mathrm{mm}$ unit, as shown by line marker at the paper. Following S I test, TBUT examination were performed by using preservative free sodium fluoresce in $2 \%$ stripe and one drop of irrigation water were instilled in the inferior fornix. Subjects that are consist of 23 female and 3 maen were allowed to close the eyes, then positioned in front of the slit-lamp biomicroscope. Once optimal position and lighting in cobalt blue filter is obtained, then subject were instructed to open the eyes, and TBUT were evaluated in second unit.

Subject were instructed to feel comfortable enough to open the eyes for corneal staining evaluation. Corneal staining evaluation uses score protocol from National Eye Institute which divided corneal area into 5 region and certain grading score by its fluoresceinstain. Regression test using SPSS software was used to analyze the correlation between each variable.

\section{RESULT}

Subjects have been using topical topical antiglaucomatous medication containing BAK for at least 3 months and also artificial tears containing sodium chloride, kalium chloride, and polyquaternium-1 (PQ-1).The more BAK is used, the lower TBUT value and vice versa. The effect of the amount and duration of BAK used is significantly correlated with TBUT $(\mathrm{p}=0.004)$. The more BAK use, the lower S I test value and the other way around. The effect of the amount and duration of BAK used is significantly correlated with S I test $(\mathrm{p}=0.043)$.

The more BAK use, the highest corneal staining score. The effect of the amount and duration of BAK used is significantly correlated with corneal staining $\operatorname{score}(\mathrm{p}=0.043)$. This study also has $\mathrm{R}$ value as $0.901 ;>0.05$, shows the 
International Journal of Psychosocial Rehabilitation, Vol. 24, Issue 02, 2020

ISSN: $1475-7192$

amount and duration of BAK used is strongly affect TBUT, S I test and corneal staining score. $\mathrm{R}^{2}$ value is 0.812 ,indicatesthat the amount and duration of BAK used is able to predict the incident of OSD as $81.2 \%$.

\section{DISCUSSION}

The result of this study found than TBUT was significantly reduced from baseline by preserved carteolol $(\mathrm{p}<0.05)$, and non-preserved carteolol usage shows no significant change in TBUT. Another study examined 40 subjects, divided into 2 groups of using anti glaucomatous containing BAK for more and less than 20 weeks, it was found that there were significant correlations between duration of therapy with Schirmer test $(\mathrm{p}=0.002)$,TBUT $(\mathrm{p}=0.004)$, Ferning test $(\mathrm{p}=0.035)$, also correlated with incidence of dry eye syndrome. Another study evaluated 30 subjects using latanoprost containing BAK with abnormal values of tear osmolarity, corneal fluorescein staining, TBUT, and subjective discomfort then switched the therapy with tafluprost preservative free, and reexamined the subjects after 6 and 12 weeks, the result showed tear osmolarity decreased, TBUT increased and discomfort decreased significantly $(\mathrm{p}<0.005)$ (Januleviciene, Derkac and Grybauskiene, 2012).

BAK has detergent molecule, which disrupts lipid layer of bacteria, so it works as preservative but also disrupts lipid layer of ocular surface and lipid layer of tear film. This effect induces inflammation by increasing tumor necrosing factor(TNF), interleukin (IL)-1,5IL-10, IL-2 and C-reactive protein (CRP)(Georgiev et al., 2011; Kaštelan et al., 2013; Artini et al., 2018).Disrupted tear lipid layer causing TBUT to decrease, and the layer below, the aqueous layer, tends to have more evaporation. On the other hand, inflammation on ocular surface also increase tear osmolarity and corneal epithel neurotoxixity then induces vicious scircle on lacrimal gland causing decreased aqueous production of tear film and those mechanism could alter the S-1 test(Rosin and Bell, 2013; Marques et al., 2015; Fidalia, Bahar and Rizki, 2019).

Some studies showed that BAK has no significant effect for Schirmer test, corneal esthesiometer and subjective complain as noted with ocular surface disease index (OSDI) quesionaire (Mathews et al., 2013). On the other hand, our study result was in line with several previous studies as described by Matthews et al. in his study, using 123subject consist of glaucoma subject and glaucoma suspect with topical medication of glaucoma showed that corneal staining significantly higher in glaucoma subjects due to number of medication ( $<<0.001)$ (Mathews et al., 2013). Previous study assed 30 healthy subjects to compare the tolerance of $2 \%$ carteolol with and without preservatives in short term use. Examination were performed before, 30,60,180minutes and 3 days of after presertative treatment. After 5 days washout, examination were performed with the non preservatives drug.

Disrupted tear film lipid layer increases tear evaporation and affect the protection function of tear film, causing irregularity and disepithelization of cornea and shows positive stain with $2 \%$ fluoresce in(Walimbe et al., 2016).All subject has been also using artificial tears with sodium and kalium chloride and PQ-1. PQ-1 itself has toxicity effect to ocular surface, despite the effect is not as great as BAK, indicates currently, this using of artificial tears has not covered the ocular surface disease issue regarding the usage of ant glaucomatous medication containing BAK(Shaarawy et al., 2014).

\section{CONCLUSION}

In this study it is concluded that the amount and duration of BAK has strong correlation on ocular surface disease manifestation, as TBUT, S I test, and corneal staining score. 
International Journal of Psychosocial Rehabilitation, Vol. 24, Issue 02, 2020

ISSN: $1475-7192$

\section{REFERENCE}

[1] Amparo, F., Schaumberg, D. and Dana, R. (2015) 'Comparison of Two Questionnaires for Dry Eye Symptom Assessment: The Ocular Surface Disease Index and the Symptom Assessment in Dry Eye', Ophthalmology, 122(7), pp. 1498-503.

[2] Artini, W. et al. (2018) 'Comparison of the goblet cells' density and the quality of tears in treatment with sodium hyaluronate $0.1 \%$ benzalkonium chloride preservative-free eye drops for patients with glaucoma and dry-eye syndrome: A double-blind randomized clinical trial', J of Physics, 51, pp. 10-16.

[3] Fidalia, Bahar, E. and Rizki, S. (2019) 'Correlation between the Use of Topical Anti Glaucoma Containing Preservative Benzalkonium Chloride with the Degree of Dry Eye Syndrome in Mohammad Hoesin Hospital Palembang', J of Res in Med and Dental Sci, 7, p. 4.

[4] Georgiev, G. et al. (2011) 'Surface chemistry study of the interactions of benzalkonium chloride with films of Meibom, corneal cells lipids and whole tears', Invest Ophthalmol Vis Sci., 52(7), pp. 4645-54.

[5] Halasz, K. et al. (2019) 'Micro/Nanoparticle Delivery Systems for Ocular Diseases', Assay and Drug Development Technologies, 17(4), pp. 152-166.

[6] Januleviciene, I., Derkac, I. and Grybauskiene, L. (2012) 'No Effects of preservative-freetafluprost on tear film osmolarity, tolerability, and intraocular pressure inpreviously treated patients with open-angle glaucoma', Clin Ophthalmol, 6, pp. 103-109.

[7] Kaštelan, S. et al. (2013) 'How ocular surface disease impacts the glaucoma treatment outcome', Biomed Res Int, 2013, pp. 1-7.

[8] Leung, E., Medeiros, F. and Weinreb, R. (2008) 'Prevalence of ocular surface disease in glaucoma patients', J Glaucoma, 17, pp. 350-355.

[9] Marques, D. et al. (2015) 'Lacrimal osmolarity and ocular surface in experimental model of dry eye caused by toxicity', Bras Ophthal, 12(2), pp. 57-59.

[10] Mathews, P. et al. (2013) 'Evaluation of ocular surface disease in patients with glaucoma', Ophthalmology, 120(11), pp. 2241-8.

[11] Moss, S., Klein, R. and Klein, B. (2000) 'Prevalence of and risk factors for dry eye syndrome', ArchOphthalmol, 118, pp. 1264-1268.

[12] Rosin, L. and Bell, N. (2013) 'Preservative toxicity in glaucoma medication: clinical evaluation of benzalkonium chloride-free 0.5\% timolol eye drops', Clin Ophtalmol, 7, pp. 2131-35.

[13] Shaarawy, T. et al. (2014) Glaucoma. 2nd edn. Elsevier Saunders.

[14] Walimbe, T. et al. (2016) 'Effect of benzalkonium chloride-free latanoprost ophthalmic solution on ocular surface in patients with glaucoma', Clin Ophthalmol, 10(215-17).

[15] Zhang, X. et al. (2019) 'Ocular surface disease andglaucoma medication: a clinical approach', Eye Contact Lens, 45(1), pp. 11-18.

Table 1: Effect of BAK on TBUT

\begin{tabular}{c|c|c|l|l|c}
\hline Parameter & $\begin{array}{c}\text { The } \\
\text { lowest }\end{array}$ & $\begin{array}{c}\text { The } \\
\text { highest }\end{array}$ & Mean & $\begin{array}{c}\text { Standard } \\
\text { Deviation }\end{array}$ & $\begin{array}{c}\text { Regression } \\
\text { Test }\end{array}$ \\
\hline $\begin{array}{c}\text { Total BAK } \\
\text { amount (mcg) } \\
\text { TBUT (second) }\end{array}$ & 558 & 58692 & $1.23 \times 10^{4}$ & 13334.16 & $0.004^{*}$ \\
\hline
\end{tabular}

Table 2: Effect of BAk on S I Test

\begin{tabular}{l|c|c|c|c|c}
\hline Parameter & $\begin{array}{c}\text { The } \\
\text { lowest }\end{array}$ & $\begin{array}{c}\text { The } \\
\text { highest }\end{array}$ & Mean & $\begin{array}{c}\text { Standard } \\
\text { Deviation }\end{array}$ & $\begin{array}{c}\text { Regression } \\
\text { Test }\end{array}$ \\
\hline Total BAK & 558 & 58692 & $1.23 \times 10^{4}$ & 13334.16 & $0.043^{*}$
\end{tabular}


International Journal of Psychosocial Rehabilitation, Vol. 24, Issue 02, 2020

ISSN: $1475-7192$

\begin{tabular}{c|c|c|c|c|c}
\hline $\begin{array}{c}\text { amount (mcg) } \\
\text { S I test (second) }\end{array}$ & 1 & 9 & 4.51 & 2.01 & \\
\hline
\end{tabular}

Table 3: Effect of BAK on Corneal Staining

\begin{tabular}{c|c|c|c|c|c}
\hline Parameter & $\begin{array}{c}\text { The } \\
\text { lowest }\end{array}$ & $\begin{array}{c}\text { The } \\
\text { highest }\end{array}$ & Mean & $\begin{array}{c}\text { Standard } \\
\text { Deviation }\end{array}$ & $\begin{array}{c}\text { Regression } \\
\text { Test }\end{array}$ \\
\hline $\begin{array}{c}\text { Total BAK } \\
\text { amount (mcg) } \\
\begin{array}{c}\text { Corneal staining } \\
\text { score }\end{array}\end{array}$ & 558 & 58692 & $1.23 \times 10^{4}$ & 13334.16 & $0.014^{*}$ \\
\hline
\end{tabular}

